\title{
Restoring Relationships, Healing Trauma, and Creating Securely Attached Classrooms
}

\author{
Chloe Drulis \\ Pepperdine University
}

Taylour Matz

Carly Samuelson

Louis Cozolino

Pepperdine University

\begin{abstract}
Our current educational system is modeled after the mass production of industrial factories, characterized by individualism, standardization, and competition. The industrial model, while efficient, largely disregards that learning is an interpersonal process. Social connection and emotional attunement activate our neurobiology for learning, while anxiety, isolation and stress inhibit those same processes. This is because our brains evolved to learn in the context of kinship groups often called tribes. This paper offers scientific support for creating classroom cultures of social cohesion which optimize students' social, emotional and cognitive development. We will also present practical applications for teachers that can be used to create tribal classroom environments within the parameters of a modern standardized curriculum.
\end{abstract}

Keywords: social neuroscience, attachment, interpersonal neurobiology, education

\section{INTRODUCTION}

Our brains are social organs, shaped by experience through a modification within the brain generally referred to as neuroplasticity. When children come from a home environment that is loving and supportive, they arrive at school with brains primed to learn, play and explore. The student whose brain has been wired to survive in a dangerous home or community environment struggles to learn in survival mode. To reach these students, physical and emotional safety must be established in order for their brains to become available for learning. As teachers, we can regulate and shape the brains of our students to enhance their ability to think, learn, and act in more thoughtful and productive ways by creating a classroom environment that is safe, secure, and supportive.

In addition to being shaped by our environments, our brains are also regulated by our significant attachment relationships. Children aren't born with an innate ability to protect themselves from danger and regulate their own fear and anxiety, so they rely on adults to make their worlds safe and teach them how to cope with stress. A child's attachment style, either secure or insecure, is first established by the nature of a 
child's relationship with their parents and caretakers, which will then unconsciously guide their feelings, attitudes, and behavior towards other adults, including teachers. This can manifest in a variety of scenarios. A child whose parents provide consistent care is likely to have positive associations with adults and feel trust and deference for their teachers. However, a child with parents who are dismissive, absent, or abusive may project distrust and poor boundaries onto their teacher, resulting in withdrawal or oppositional behavior. Worst of all, insecure attachment styles in both teacher and student can result in a fractured relationship and a young mind closed to learning. When a teacher is able to work through this resistance and form an emotional bond with a struggling student, they have the power to modify their attachment for the better within the classroom. The nature and quality of our relationships with our teachers can play a critical role in our ability to learn, understand, and internalize what we are being taught.

ATTACHMENT
The core of attachment is the ability for
babies to use the presence of their caretakers
as a way of regulating anxiety and fear. As
our brains evolved into social organs,
humans' ability to use relationships with
significant others to manage anxiety has
expanded beyond the parent-child
relationship. In the classroom, secure
attachment relationships between teachers
and students optimize learning by
regulating anxiety, stimulating motivation,
and enhancing neuroplasticity.

Students who face chronic stress demonstrate a decrease in the three building blocks of learning something new: concentration, attention, and memory. If a child goes to school thinking about problems at home that they're unable to solve, they will experience higher sustained levels of cortisol, the chemical that keeps us preoccupied with survival as opposed to feeling relaxed and ready to learn. Neuroplasticity is also inhibited during heightened states of arousal and anxiety, making it nearly impossible to learn in the classroom. Many teachers, especially those who grew up in nurturing homes and safe relationships, misinterpret their student's behavior as a personal affront rather than an expression of emotional dysregulation, fear, and defense. This heightened state of arousal is a breeding ground for violence, misbehavior, and truancy. In these situations, teachers must learn to provide students with the appropriate tools to re-regulate their emotions, get their brains back online, and reinforce to the child that they are capable of learning.

Learning is a shared experience that occurs in the context of relationships and shapes not only what we know but how we understand the world and feel about ourselves. The interdependency between relationships, optimal brain development, and learning was established as humans evolved within tight-knit tribes. The conditions that allowed us to survive in tribes, such as group cooperation and a need to belong, were transcribed into the biology of neuroplasticity. The most effective teachers in the tribe were those who mixed attention, affection and caring with knowledge and skills necessary for survival. In contrast to the one-size fits all model of industrial education, each member of the tribe was encouraged to find their unique way of contributing to the survival of the group. These social instincts still exist within each of us today. They are the same interpersonal emotions leveraged by tribal educators that have the ability to promote engagement and learning. When students come to school with a sense of safety and love, they may be able to perform well in the more factory-based model. If they come to school stressed, afraid, and alone, traditional tribal methods of learning may be necessary to create the state of mind that allows for learning to occur. 


\section{A STUDENT'S PERSPECTIVE}

Before seven-year-old Andre opens his eyes in the morning, he is jarred awake by the sound of his parents arguing in the next room. Overwhelmed by the stress of working multiple jobs and struggling to pay rent, they have been yelling a lot lately, at each other and at their four kids. For Andre, this means he has to take care of himself most of the time, and this morning is no exception. Andre has a stomachache again, something he's become accustomed to but doesn't dare bother his parents with. He's learned that by staying quiet, he can avoid the emotional landmines that now characterized his interactions with his parents. They are so distracted that they don't even notice the younger children fighting over toys in the living room, so Andre takes it upon himself to play referee and in the process, his five-year-old brother punches him in the arm. Unfortunately, he has become used to being the adult at home, and as a result, his siblings often take out their fears and frustrations on him.

NEUROSCIENCE
Through millions of years of social evolution, our
brains, minds, and our ability to connect with
others have been woven into a complex
biopsychosocial learning system, linking our
ability to learn with a sense of safety, connection,
belonging, and purpose.
Our ability to learn relies on our brain's ability to
change and adapt in response to a new experience
through a diverse set of processes called
neuroplasticity. In recent years scientists have
discovered that the biological and genetic
processes driving neuroplasticity are largely
regulated by neural systems dedicated to
emotional regulation, bonding, and attachment.
These connections highlight the contrast between
the social-emotional learning context of
traditional tribal life with what a student
encounters within a modern classroom. Creating
a tribal classroom is an attempt to develop social
and emotional workarounds which leverage the
biology and genetic processes which mediate
learning to optimize classroom learning.

By the time he gets to school at 8 a.m., he has expended as much emotional energy as most children his age have to use in a week. Andre used to love school, but his teachers have been getting upset with him for not paying attention in class, so he's started dreading it. That is, except for Mr. Dante's history class. In the presence of this warm and gregarious teacher, the knots in his stomach begin to untangle. Mr. Dante was raised by a loving single mother who worked full time and was the sole caretaker of his ailing Grandmother. Consequently, he spent a lot of his time in an after-school drama program where he bonded with a teacher who would eventually inspire his own career. Mr. Dante's role as an educator has always meant more than homework and lesson plans, and he takes great pride in the connection that he fosters with each of his students. 
Andre is transported by his animated storytelling and can get a glimpse of the world that exists beyond the turmoil of his present life. To his own surprise, he finds himself eagerly asking questions and reflecting on details of the lesson for days afterward. Mr. Dante noticed early on that Andre was highly sensitive to the emotions and needs of others and leveraged these innate qualities to cultivate something Andre lacked: the ability to be sensitive to his own needs and emotions. Mr. Dante assigned him to work with another peer as the "conflict managers," where they team up to facilitate problem solving among peers. Andre has plenty of practice at home taking care of others, and in Mr. Dante's class, his abilities are valued by both his teachers and peers, which fosters a sense of pride and connectedness.

Andre leaves Mr. Dante's class feeling a sense of purpose and connection, but this quickly dissolves in the face of the rigid demands of Ms. Roger's math class. Ms. Rogers is passionate about the value of building math skills in young minds. For her, math had been the ticket out of her tumultuous home life when a university scholarship allowed her to escape her verbally abusive father. The predictability of math provided a reprieve from constant parental instability and her aptitude prompted rare displays of praise from

her parents. Ms. Rogers teaches math the way it was taught to her, with a rigid structure and undertone of competition which she has come to believe fosters motivation for learning. Following a strict protocol, she gives daily timed quizzes to students and gives star stickers to high-performing children.

In this classroom environment, Andre struggles to maintain focus while he completes a series of worksheets alone at his desk. He becomes keenly aware of his neighbor, who is speeding through the assignments at twice his pace, which only makes Andre anxious and more distracted. Andre watches as several classmates receive stars in front of the class for completing the work and it is evident he won't finish on time. Discouraged and running out of emotional energy, Andre gives up and puts his head down on his desk. Ms. Rogers becomes triggered by what she perceives as a personal affront and chastises Andre in front of the class. This interaction serves to strengthen the pattern of emotional dysregulation between them that will continue throughout the semester. In contrast to his experience of Mr. Dante's class, his interactions with Ms. Rogers create a state of brain and mind that makes it impossible for Andre to attend, concentrate, and learn.

While he will most likely be labeled as having ADHD or suffering from emotional difficulties, Andre's challenges may be better understood through the dynamic relationship between stress, neuroplasticity, and learning. Andre most likely enters school with a brain that is essentially "turned off" to learning due to the high level of arousal, stress, and agitation (remember his stomach aches) he endured before he left home. The sensitivity of Andre's brain to his social emotional context is illustrated by his positive adaptation to the atmosphere of Mr. Dante's classroom, which is characterized by connection, emotional support, and collaboration. These conditions downregulate Andre's arousal and create a state of brain and mind which allows him to engage, attend, and learn. A state which quickly changes when he enters a situation where he received little emotional connection or support in the context of individual competition. The fact that children react differently across situations is often missed by teachers and administrators, so they tend to be labeled with some internal problem rather than as reacting to changes in their environments. In the next section of this paper, we will discuss the scientific theories and discoveries that make a safe and secure relationship such a powerful tool for brain-building and learning.

\section{THE CORE TENETS OF A TRIBAL CLASSROOM}

The human brain evolved into its modern form during a time when tribal culture was the standard for living, and the fundamental interdependence of group members shaped each brain into a nexus of social connectivity. The same chemistry, biology, and wiring that we have today were established during our long prehistory. The tribe was united by bonds of kinship, culture, and mutual survival. Throughout history, these groups were an extended family. They cared and looked out for one another and fostered a sense of connection that increased their adaptability and chances of survival. Our tribal history is relevant to modern education because we are biologically wired to live, learn, and thrive most optimally when embedded in a group. By adapting the core tenets of tribal learning into a modern classroom, teachers are able to tap into the social instincts that activate learning, exploration, and growth. 


\section{TRIBAL CLASSROOM \\ A tribal classroom activates the social and emotional processes which stimulate neuroplasticity and learning.}

We've identified four core tenets that tribes relied on in order to function most optimally. The tenets promote relevant education, the assigning of roles, cultivating a strong emotional connection to one another and the use of storytelling as a mechanism for learning. By applying these principles to the modern classroom, teachers have an opportunity to build a cohesive social group that fosters connection and encourages learning.

\section{Learning Happens in the Context of Real Life}

As a member of a tribe, the motivation to learn was innate. Lessons passed intergenerationally were the vehicle one used to navigate a harsh world. There were no consistent curriculums because educators were always adapting to new environments, changing circumstances, and the needs of group members. In order for a tribe to effectively operate, teaching had to be flexible--always adapting the material in support of a larger learning opportunity that would encourage students to think critically about their role within the community and how they could be most contributory. The education also had to be relevant to their lives. We evolved to learn while moving through the natural environment in ways that simultaneously support exploration, neuroplasticity, attention, and memory. Tribes understood the importance of integrating education with real-life applications in order to support technical learning as well as the skills needed to navigate their lives.

\section{Everyone Has a Role}

Men, women, and children all contributed to the well being of the tribe, each playing an integral role with its own unique purpose. As a whole, the tribe had common interests to strive for and common enemies to fight against. It was essential that a group was devoted to understanding how each group member could support and protect one another, particularly the young, who needed to feel both motivated and safe; a neuroplastic "sweet spot" where energy can be channeled towards learning. These roles were established in order to ensure that the tribe would thrive, but they also promoted individual purpose and satisfaction. By honing individual strengths and weaknesses, the group boasted a diverse profile of members who functioned well together and leaned on one another. This process of identifying what individuals were good at was reinforcing, confidence-boosting, and encouraged connection and trust within the group. It also promoted a communal way of thinking about oneself as a piece of a larger whole.

\section{Emotional Bonds of Connection}

As illustrated in the vignette about Andre and Mr. Dante, we learn best from people we care about and who care about us. This is a key tenet of tribal life and one that can be understood particularly well through the symbiotic relationship between teacher and student. Teachers were most often older members of the tribe who were trusted and respected, making it natural for them to impart wisdom on the young minds of the group. By teaching from a place of care and mutual respect, students learned social skills, compassion, and empathy, while still fostering cognitive intelligence and instilling a healthy respect for authority. The emotional connections developed within the classroom were reinforced in all other aspects of tribal life, as teachers were also mentors of the students outside of class, giving them an added opportunity to model the behaviors that they teach. Teachers were also highly motivated to educate students about how best to function well in the world because it enhanced the survival rate of the whole group. Each relationship was mutually beneficial. 


\section{Learning Through Stories}

Tribal teaching revolved around storytelling. When the tasks, challenges, successes, and failures of the day came to a close, the tribe would gather to tell their stories. It served a dual purpose: education and preservation. The academic side of storytelling involved teaching essential lessons, and the preservation component involved the passage of knowledge and history. It was a creative method of educating young people about the risks and rewards of life. It also aligned with the way that the human brain processes and learns new information. What tribal members knew intuitively was that we have a fairly limited memory capacity for isolated pieces of information like words and numbers. On the other hand, we appear to have an unlimited capacity to remember stories and songs because these are the kinds of memories upon which preliterate people rely on. By simultaneously engaging multiple executive and memory systems at the same time, storytelling (and listening) supports the brain's internal communication processes. Socially, stories forced tribal members to put feelings and experiences into words, encouraged thoughtful reflection about their experiences, and prompted them to consider the perspective, experience, and knowledge of the listener.

\section{Summary}

Techniques like these could be called tribal "workarounds" within a modern classroom. The act of drawing on principles of our basic social instincts to connect and attune creates a better learning environment. It also supports emotional regulation by attempting to make each student feel seen, heard, felt, and accepted into the group. Assignments aim to integrate the students' lived experiences into the classroom and challenge them to take an active role in their own stories with the support of the members of their new tribe. By stepping foot into their world, educators have an opportunity to show their kids that not only are they unafraid of their struggles, but they also want to know more. In doing so, it facilitates a kind of trust in the classroom that could dismantle their defenses against learning. The sense of belonging and emotional support fostered by shared experiences alter the emotional dynamics of the classroom and create a state of brain and mind which allows them to be open to learning.

\section{AN INSIDER'S TAKE: THE PREDICAMENT OF THE MODERN TEACHER}

2020 was a year that had many of us questioning our institutions and reexamining the very purpose of our lives; we scraped away all that felt extraneous in order to discover what was truly essential. Those of us in the education system felt this acutely. A former colleague and friend told me ( $\left.{ }^{\mathrm{TM}}\right)$ that every morning on her way to the high school where we taught, she would have to pull over to throw up on the side of the road. The pure dread of teaching in an environment that did not align with her instincts or her values caused this powerful, visceral reaction. This goes far beyond burnout; it is making us unwell. She did not teach Physics or Calculus, but Culinary Arts, a position that one would assume would be filled with creativity and joy rather than anxiety to the point of nausea. She was one of the best, most beloved teachers at that school, but she ended up leaving the classroom in search of a more nurturant teaching and learning environment.

I soon found myself in the same predicament. Instead of feeling a deep sense of community, collaboration, and creativity within my classroom, I was struggling to stay afloat, adhering to every standard, mandate and directive my school site gave me. I desperately needed a way out, a way to rediscover the true nature of teaching and learning. I yearned to find the validation that my educational instincts were correct, and I soon found myself at Harvard working on a Master's degree in education. There, as I delved deeper into my studies, my concerns of industrialized schooling continued to grow. Students who are privileged enough to have resources available to them, find themselves as silos in an educational environment that is based on competition, perfect grades and test scores and getting into top universities. Still, they feel unseen for who they truly are, even if society grants them the gold star of "success." It is an even steeper uphill battle for our students in underserved communities to find an alternative method of learning that inspires them and honors their backgrounds and stories. 
For many educators, knowing that a child comes from a difficult home, dangerous community environment, or both, can create feelings of pessimism and hopelessness. The social problems appear too immense, and the teacher has so little power to influence these broader social issues. The good news is that we have more power than we think. The first step is to understand that creating a quality relationship with one's students is just as important to teaching the curriculum, and by attuning to their needs and remaining mindful of the way that humans evolved to learn, we are making it possible for them to overcome the obstacles that have stood in the way of their education. A caring and secure classroom can be an oasis providing students a safe haven from their stressful worlds and the opportunity to develop an identity as a learner and agent of change in their community.

\section{The Teacher as Guide and Mentor}

Tribal educators have a unique opportunity to prune the harmful effects of industrialized education and plant the seeds of learning as our ancestors once did. The Tribal Classroom is a healing-centered classroom, and the healing must begin with the teacher. Like most mammals, humans learn first by imitating their parents and other elders. Imitation also remains a central mode of learning throughout life. Because of this, there is nothing more important for a teacher than engaging in his or her own healing process prior to entering the classroom. It would be immensely valuable for credentialing programs to integrate a variety of mental health supports and healing modalities into their programs. Once in the classroom, tribal teachers will need to continue their own healing journey, whether that be therapy, support groups, spiritual engagement, somatic work, or another method of inner transformation and personal growth.

Educators with unexamined internal lives or unresolved trauma are at risk of being triggered by students' behaviors in ways that exacerbate their struggles with learning. Negative emotions are both contagious and synergistic. The trauma of a student can easily trigger the traumatic emotions in each other and their teachers (Think back to the diverging experience of Andre and Ms. Rogers). When a teacher has the awareness to self-regulate when faced with a student's triggered behavior, they have the opportunity to deescalate the situation rather than reacting in a punitive manner. Modeling appropriate ways to respond to stress and conflict, establishing healthy relationships, and appropriate boundaries are essential tools for creating a safe and secure classroom, and activate the brain and mind to learn. It takes the whole teacher to educate the whole child.

The social brain has evolved to learn from people with whom we share warmth, connection, and trust. When a tribal teacher occupies the center of emotional gravity of the classroom, the opportunity for this same secure connection and openness becomes possible. It might sound radical at first, but love and hope are powerful but underutilized tools to enhance learning, especially those students who have experienced trauma. Paulo Freire, bell hooks, Shawn Ginwright, Jeff Duncan-Andrade, and other esteemed educators bring love and hope to their classrooms. They are also proponents of educators engaging in their own healing to reintegrate their mind, body, and spirit to best guide their young people. Author, professor, and activist, Shawn Ginwright, stresses the importance of a teacher's personal healing in his book Hope and Healing in Urban Education: How Urban Activists and Teachers are Reclaiming Matters of Heart:

Implementing practices that facilitate healing begins with healing yourself [the teacher]. It is not possible to create vibrant, thriving classrooms, schools, and community programs for young people with adults who are toxic! Implementing these practices requires that we integrate a philosophy and worldview of well-being, healing, and joy into our practices, and only then will we experience "successful" implementation. (Ginwright, 150)

Teachers are encumbered with a litany of challenges from the political and bureaucratic to the personal and financial. The first step to radical change in these areas is for these educators to become curious about their own internal landscape, their triggers, fears, hopes, values, and purpose. If educators are to be guides and mentors, aspirational people to whom our young people look up to, self-actualization is a necessary component to the classroom as a whole, a place where both teachers and students have the ability to grow into their highest selves. The layout and feel of their classroom, the lessons they create or, in many cases, 
"tweak," the literature they offer, the personal stories they choose to tell, the assignments they assign, their level of interest and involvement in their students' extracurricular activities and family lives, are all invitations to curate a learning environment that is hopeful, loving, and healing-centered. This curation brings a sense of soulfulness back into the lives of students and teachers alike.

\section{TWO TOOLS FOR CREATING A MODERN TRIBAL CLASSROOM}

As important figures in the lives of their students, teachers can become practitioners of hope and healing. They can pull from a variety of practices that enable care and healing to be the core of their pedagogy. Further, the science suggests that this social-emotional context will enhance the abilities of their students to learn the curriculum. Inviting students to examine their internal world is an integral part of development. The path to self-understanding can be enhanced through two practices that have their roots in ancient indigenous culture, mindfulness and Circle Process. It is, however, important to have training and practice in both of these modalities with experienced and reputable teachers before bringing them into the classroom.

\section{Mindfulness and Meditation Practices}

Mindfulness meditation helps students to be aware of and regulate their emotional and mental state, as well as to sit in non-judgment of the present moment. With so many external pressures and expectations young people face on a daily basis, having time dedicated every day to an internal, contemplative practice helps them to more clearly see who they are at their core. Mindfulness is, therefore, not just a practice to begin the day but the essence of how a tribal classroom functions. The feeling of "you matter" is an essential component to the learning of all young people, and the first step of that is figuring out who exactly that "you" is and strengthening the ability to sit with oneself no matter how you are feeling or what you are experiencing.

Mindfulness or other meditative practices are an invitation, never a requirement. If students do not wish to participate, the teacher or facilitator may ask that for the duration of the practice, cell phones, and other electronics be put away, and those who have opted out have the choice to lay their heads down, color, draw, read, or partake in another silent activity, as to respect those who are practicing the mindfulness. Lights can be turned off or dimmed; if possible, lamps and battery-operated candles can add a soothing ambience to the classroom. The changing of the lights from bright fluorescents to a softer hue signals to the students that the atmosphere and tone of the room will be changing and supports their nervous system to follow suit. A singing bowl or other pleasant, auditory bell is helpful to both start and end the practice. If the teacher is comfortable enough to facilitate the practice, they may want to guide the students to close their eyes, but only if that feels safe for them.

Mindfulness techniques often suggest that the attention of the practitioners be on the sensation of their breath; however, this may not be suitable for students who have a trauma history, as heavy or labored breathing could have been activated during the traumatic event. In this scenario, it may be better for those students to have their attention on other sensations such as sounds or the feeling of support by the chair or ground. An ideal position for the practitioners to be in is sitting near the front of the chair, feet flat and rooted on the ground, knees slightly apart, hands in a comfortable position, either face up or down, or folded in the lap. The spine should be upright but not uptight, like a queen or a king sitting on one's throne.

Doing a body scan can be helpful to allow students to settle into their bodies and to bring awareness to how they are feeling both physically and mentally. Again, it is important for students to be assured that all feelings of body and mind are welcome, natural, and normal. A tribal teacher will have a sense of the appropriate length of mindfulness practice for their group of students. Elementary school-age can usually do about three to five minutes; middle school and high school students can usually do about ten minutes. However, it is always better to start in small, manageable chunks for all young people. 


\section{Circle Process}

Where mindfulness focuses on the internal landscape, Circle Process brings this awareness into the public sphere, where participants are encouraged to tell their stories. In her book The Little Book of Circle Processes: A New/Old Approach to Peacemaking, Kay Pranis writes:

Peacemaking Circles use structure to create possibilities for freedom: freedom to speak our truth, freedom to drop masks and protections, freedom to be present as a whole human being, freedom to reveal our deepest longings, freedom to acknowledge mistakes and fears, freedom to act in accord with our core values. (Pranis, 11)

Participants sit in a circle, either in chairs or on the floor. If desired, objects that hold meaning for the group are arranged in an altar and placed in the center of the Circle. This helps to bring attention to the group's shared values and common interests. A Keeper or facilitator is the person who holds the space for participants and is tasked with ensuring that everyone feels safe and that each voice that wants to speak is heard. A Talking Piece is another essential element to the Circle. This is any small object that holds some significance, like a special rock, feather, crystal, etc., that is passed around the Circle. Whoever has the Talking Piece has permission to speak, but it is never required that someone talk. Sometimes, a Circle is opened with a few moments of silence or meditation to bring focus to the group and to signal the transition from one state of being into another. If this is the first time a group is meeting in a Circle, agreed-upon values and guidelines should be established to ensure safety, courage, and respect. Circles are a place where the soul can be free to be its true self and may create the first and only place a young person has permission to express themselves without judgment and the opportunity to learn the skill of self and co-regulation. Beginning class each day in a Circle allows soul freedom to set the tone for the lifespan of the learning community.

Trust, vulnerability, and specific, agreed-upon values within the group, are the essence of Circle Process. The act of ritual or ceremony is a powerful mechanism to ensure the Circle does what it intends to do. Whether the Circle is to make peace or amends, to heal wounds, resolve conflict, build community, sentence, reintegrate, or celebrate, ritual is the method of actualization. When looking at this through the lens of neurobiology, everyone in the Circle will have the opportunity to experience emotional resonance with the story of another, thereby helping each other to co-regulate, bringing about feelings of safety and trust, and activating their brains and bodies to learn.

Both mindfulness and Circle Process can be integrated into the secular, tribal classroom. In our busy, modern world, we know how important it is to have a balanced sense of self before we are able to heal and offer our energy to the group. Additionally, mindfulness practice and Circle Process are two of many modalities that can help heal the wounds brought on by industrialized education by intentionally acknowledging and holding the orphaned spirit of both teachers and students alike.

\section{Curriculum the Tribal Way}

I $\left({ }^{\mathrm{TM}}\right)$ have a student who loves to cook. Building on his interest, or any interest a young person has, and embedding it directly into the curriculum is one example of how an educator can bring tribal education into the modern classroom. By attuning to their students' propensities, a tribal teacher will recognize this as an opportunity to foster trusting relationships, while also adhering to curriculum standards. Utilizing food and cooking as the lens, the initial step is to invite the students into the conversation of how to make food part of their learning; this sense of agency is a significant cornerstone of the tribal classroom, as it honors their ability to make decisions about their own educational journey, and allows them to be seen and respected.

From this experience, a discussion ensued that led to class deciding that science and chemistry standards could be met by implementing lessons on the science of cooking, sustainability, the negative effects of food waste, and learning about locally grown and raised foods. From this point, the conversation circled around reflections on food insecurity and other social justice issues related to poverty, health, and 
body image. To meet English standards, writing a food or restaurant review would serve as a lesson on how to form an opinion and backing that opinion with solid reasoning and evidence. The core topic of food, a central concern of tribal life, can be linked to multiple streams of learning. Recognizing and being led by students' interests helps them gain a sense of agency and can lead to a richer, deeper, and more integrated learning experience.

Along with developing lessons around my students' individual interests, I also find it vital to meet them where they find challenges. At the beginning of each school year, I have my students analyze the poem The Rose that Grew From Concrete by Tupac Shakur (I must give credit to Jordana Henry, my esteemed colleague who created this lesson, a tribal teacher in her own right). Tupac is a great connector, a poet, and a lyricist, who will probably never lose his relevancy when testifying about the struggles of oppression and violence. He is a portal into the world of my students and is a bridge between what they are already familiar with to a new world of discovery. After we analyze the poem, I draw a rose in concrete on the board. We do a class brainstorm of all the struggles and challenges we face in our community. This year our concrete consisted of gang violence, COVID, homelessness, drug and alcohol abuse, food insecurity, distance learning, income inequality, police brutality, racism, health issues, and citizenship status.

Above the concrete, where the rose is growing, is the fresh air, and here we end on a hopeful note and brainstorm the parts of our lives that keep us resilient. This year, our fresh air was: setting a life goal, friends, family, sports, art, music, entertainment, nature, and food. As I get to know the students better and we become more comfortable with each other as a group, the direction of our classroom begins to take shape, and we brainstorm ideas of how we can bring the classroom to the community. This simple, yet profound activity, helps to establish the tribal approach of teaching and learning, which is based on healthy relationships and promotes the well-being of the entire group, the small tribe of the classroom, and the larger tribe of the surrounding community. Of course, not all students are interested in the same type of outreach, but we go where the opportunities arise, and there is always a job that fits every young person. Oftentimes, a novel exposure will inspire a skeptical student and provide a rich learning experience for all of us. Based on the type of outreach we do, we do our best to select literature that aligns with the volunteer work we are involved in, to deepen and expand our learning. For example, if our outreach involves supporting those with addiction, we may read Go Ask Alice. If we are working in nature conservation we may choose to read The Call of the Wild.

Our assignments and projects are directly related to the work we are doing in the community; perhaps it's the students writing a letter of concern to community leaders, making their voices heard at town hall meetings, creating brochures, social media posts, promotional videos, and documentaries to bring awareness to a particular issue. When the young people begin to feel comfortable and settle themselves into the tribal classroom, the personal challenges they face start to come forward during the Circle Process, and they naturally begin to reflect on how these challenges impact the community at large, and the power they have to do something about it. My students have disclosed personal struggles such as gang violence, abuse, incarcerated parents or siblings, the death of a friend, addiction, homelessness, eating disorders, and mental illness. By empowering students to move to action by serving others with these same struggles, it guides them to transform their mess into a masterpiece.

Serving the community is the modern tribal classroom's way of fighting against a common enemy. Instead of spears, the fight is carried forward through personal storytelling. And the enemy is not a rival tribe or wild predators, but systems of oppression, personal struggles, and the challenges of growing up in an all too often dangerous world. Whether lessons are created from a student's individual interest or derived from a real-life issue in the community, the higher the stakes, the more intrinsically motivated the student. Not only are they deeply motivated to learn and succeed, but they are also proud of their accomplishments when recognized by their peers, which drives a deep sense of purpose and belonging a human trait woven through millions of years of social evolution.

As a high school teacher in an alternative education program, I have the unique privilege to work with a small group of students on individualized learning plans. However, any teacher of any age group 
can be a tribal educator, as the basis of a tribal classroom is nurturing and trusting relationships. Additionally, the creative workarounds are unique to each educator; we, like our students, are not merely cogs in a wheel that can be easily replaced. Utilizing your particular talents and passions will be a powerful addition to the learning atmosphere. If you are unable to design an entire unit based on one particular interest, make note of what is of general interest to your student population (music, sports, Netflix shows, animals), and weave it into their learning. Choose a list of books and have the students vote on the one the class reads, or how they want to demonstrate what they've learned; perhaps they will want to give a presentation, write an essay, create a piece of artwork, sing a song, or perform a scene. Another way to make the class more tribal is to ground the lessons and assignments in the real world. It is much more meaningful for the young people to have their work be based on some sort of community outreach, rather than merely handing in papers to their teacher. If taking the students out into the community isn't feasible, have them come to you, or give the support you can from afar. Technology, including Zoom, have made distance less of a barrier to connecting and lending support to those in need. The original tribal classroom was based on survival, and the same is true today. We may not have to worry as much about day-to-day survival (although many of my students do), but how we connect our lessons to our ability to thrive will be much more impactful for our young people than just the bare bones of standards and curriculum.

\section{CONCLUSION}

The central implication of social neuroscience to educators is this: our ability to learn is interwoven and dependent on the quality of our relationships. The level of support within teacher-student relationships unconsciously communicates the degree of safety within the classroom. Human brains, especially the developing minds of children, are unable to function without a baseline of security. A secure environment is a natural byproduct of teachers attending to the interests, challenges, and needs of individual students, as well as providing ample opportunities for students to build interpersonal bonds amongst themselves. Like a well functioning tribe, this creates a classroom culture that is cohesive, interdependent and supportive. These are also the optimal conditions to prime young brains to learn, explore and grow. Promoting mutual respect, adapting for the student's needs, and honoring who they are as uniquely different people with varying skills and interests is not only the kind thing to do; it's prescribed by the way our brains evolved to learn.

The techniques and workarounds that we've outlined in this paper are based on the way that our brains have evolved to learn. These strategies also promote cognitive development, cultivate social and emotional intelligence, and teach important life skills such as creativity, communication, and problem-solving. They empower students to discover and hone their unique abilities so that they can best contribute first to their classroom unit, and eventually to their communities and society at large. By applying the principles of a tribe to the modern classroom, educators have the power to turn on the learning networks of a student's brain, adequately preparing them for the remainder of their time within the standardized educational system while also preparing them for the interpersonal challenges of adolescence and adulthood.

The universal goal of education is to provide each student with learning and growth opportunities to realize their full potential. We propose that the current standardized model of education can be bolstered by incorporating tribal methods of teaching to enhance cognitive, social, and emotional development. When teachers emulate the teaching conditions of a tribe, they are recreating the conditions in which our brains, biochemistry, and social instincts evolved into their current form. This means that a tribal classroom optimizes neuroplasticity, bodily regulation, and prosocial behavior. We don't have to reinvent the entire educational system in order to create a tribal learning environment. A tribally minded educator's success is based on his or her ability to create a classroom that fosters learning while also checking the boxes required by the curriculum. Pedagogical strategies such as hope, love, and healing, while historically deemed as "soft skills" in our industrial model of education, are in actuality the biological and historical bedrock of neuroplasticity and learning. 


\section{REFERENCES}

Cozolino, L. (2013). The social neuroscience of education: Optimizing attachment and learning in the classroom. New York, NY: W.W. Norton \& Company.

Cozolino, L. (2014). Attachment-based teaching: Creating a tribal classroom. New York, NY: W.W. Norton \& Company.

Ginwright, S. (2016). Hope and healing in urban education: How urban activists and teachers are reclaiming matters of the heart. New York, NY: Routledge.

London, J. (1903). The call of the wild. New York, NY: Macmillan \& Co.

Pranis, K. (2005). The little book of circle processes: A new/old approach to peacemaking. New York, NY: Good Books.

Shakur, T. (2009). The rose that grew from concrete. New York, NY: Pocket Books.

Sparks, B. (1971). Go ask Alice. New York, NY: Simon Pulse. 